fetal hematopoietic and TMD cells (23). Such experimental tools would allow systematic evaluation of human cT21 in the correct developmental context.

Whether DYRK1A is functioning through the NFAT pathway or another pathway in DS-AMKL, its inhibition may have therapeutic benefit. Malinge et al. provide a proof of concept that DYRK1A inhibitors may be clinically useful in the context of DS-AMKL by demonstrating that harmine, a small-molecule inhibitor of DYRK1A kinase activity, can inhibit the growth of megakaryoblastic leukemic cell lines with trisomy 21 (2). Thus, DYRK1A inhibitors may be a specific targeted therapy for DS-AMKL.

\section{Acknowledgments}

Our research of the leukemias of Down syndrome is funded by Children with Cancer (UK), NIH, Waxman Cancer Research Foundation (New York), Israel Cancer Research Foundation (New York), Israel Science Foundation (Regular, Legacy, and I-CORE programs), Israel USA Binational Foundation, the Chief Scientist of the Israeli Health Ministry, the Clinical Genetics Foundation (Israel), and William Lawrence Blanche Hughes Foundation (USA). Y. Birger is a European Hematology Association Fellow and funded by the Leukemia Research Foundation (Chicago).
Address correspondence to: Shai Izraeli, Sheba Medical Center, Tel Hashomer, 52621, Israel. Phone: 972.3.530.5943; Fax: 972.3.530.5942; E-mail: sizraeli@sheba. health.gov.il.

1. Hasle H. Pattern of malignant disorders in individuals with Down's syndrome. Lancet Oncol. 2001;2(7):429-436.

2. Malinge $S$, et al. Increased dosage of the chromosome 21 ortholog Dyrk1a promotes megakaryoblastic leukemia in a murine model of Down syndrome. J Clin Invest. 2012;122(3):948-962.

3. Malinge S, Izraeli S, Crispino JD. Insights into the manifestations, outcomes, and mechanisms of leukemogenesis in Down syndrome. Blood. 2009;113(12):2619-2628.

4. Chou ST, et al. Trisomy 21 enhances human fetal erythro-megakaryocytic development. Blood. 2008;112(12):4503-4506.

5. Tunstall-Pedoe $O$, et al. Abnormalities in the myeloid progenitor compartment in Down syndrome fetal liver precede acquisition of GATA1 mutations. Blood. 2008;112(12):4507-4511.

6. Izraeli S. Trisomy 21 tilts the balance. Blood. 2008; 112(12):4361-4362.

7. Hertzberg L, et al. Prediction of chromosomal aneuploidy from gene expression data. Genes Chromosomes Cancer. 2007;46(1):75-86.

8. De Vita S, et al. Trisomic dose of several chromosome 21 genes perturbs haematopoietic stem and progenitor cell differentiation in Down's syndrome. Oncogene. 2010;29(46):6102-6114.

9. Salek-Ardakani S, et al. ERG is a megakaryocytic oncogene. Cancer Res. 2009;69(11):4665-4673.

10. Stankiewicz MJ, Crispino JD. ETS2 and ERG promote megakaryopoiesis and synergize with alterations in GATA-1 to immortalize hematopoietic progenitor cells. Blood. 2009;113(14):3337-3347.

11. Ng AP, et al. Trisomy of Erg is required for myeloproliferation in a mouse model of Down syndrome. Blood. 2010;115(19):3966-3969.
12. Klusmann JH, et al. miR-125b-2 is a potential oncomiR on human chromosome 21 in megakaryoblastic leukemia. Genes Dev. 2010;24(5):478-490.

13. Park J, Song WJ, Chung KC. Function and regulation of Dyrk1A: towards understanding Down syndrome. Cell Mol Life Sci. 2009;66(20):3235-3240.

14. Arron JR, et al. NFAT dysregulation by increased dosage of DSCR1 and DYRK1A on chromosome 21. Nature. 2006;441(7093):595-600.

15. Muller MR, Rao A. NFAT, immunity and cancer: a transcription factor comes of age. Nat Rev Immunol. 2010;10(9):645-656.

16. Li X, et al. Calcineurin-NFAT signaling critically regulates early lineage specification in mouse embryonic stem cells and embryos. Cell Stem Cell. 2011;8(1):46-58.

17. Ram G, Chinen J. Infections and immunodeficiency in Down syndrome. Clin Exp Immunol. 2011; 164(1):9-16.

18. Lima FA, et al. Decreased AIRE expression and global thymic hypofunction in Down syndrome. J Immunol. 2011;187(6):3422-3430.

19. Baek KH, et al. Down's syndrome suppression of tumour growth and the role of the calcineurin inhibitor DSCR1. Nature. 2009;459(7250):1126-1130.

20. Giraudier S, et al. Overexpression of FKBP51 in idiopathic myelofibrosis regulates the growth factor independence of megakaryocyte progenitors. Blood. 2002;100(8):2932-2940.

21. Ge Y, et al. The role of the proto-oncogene ETS2 in acute megakaryocytic leukemia biology and therapy. Leukemia. 2008;22(3):521-529.

22. Ozery-Flato M, Linhart C, Trakhtenbrot L, Izraeli $\mathrm{S}$, Shamir R. Large-scale analysis of chromosomal aberrations in cancer karyotypes reveals two distinct paths to aneuploidy. Genome Biol. 2011;12(6):R61.

23. Chou ST, VanDorn D, Yao Y, French DL, Weiss MJ. 2011. Patient-derived induced pluripotent stem cells reveal distinct hematopoietic defects conferred by Trisomy 21 and truncated GATA- 1 . Abstract \#911 presented at: 53rd Annual American Society of Hematology Meeting. December 13, 2011. San Diego, California, USA.

\title{
Popeye proteins: muscle for the aging sinus node
}

\section{Bastiaan J. Boukens ${ }^{1,2}$ and Vincent M. Christoffels ${ }^{1,3}$}

\begin{abstract}
${ }^{1}$ Heart Failure Research Center, ${ }^{2}$ Department of Clinical and Experimental Cardiology, and ${ }^{3}$ Department of Anatomy, Embryology, and Physiology,
\end{abstract} Academic Medical Center, Amsterdam, The Netherlands

\begin{abstract}
The electrical impulses that dictate the rhythm of the heartbeat in normal situations and during exercise or stress are initiated by a small number of sinus node pacemaker cells. Senescence and dysfunction of the sinus node affects many people later in life, causing physiologically inappropriate heart rates, but the underlying mechanisms are not well understood. In this issue of the JCI, Froese and colleagues show that deficiency in either Popeye domain containing 1 (Popdc1) or Popdc2 leads to sinus node dysfunction under stressed conditions in aged mice. The mechanism reported to underlie the effects of Popdc1/2 deficiency in mice may cause the stress-induced sinus node dysfunction found in many aged individuals and may point to new strategies for therapeutic intervention.
\end{abstract}

Conflict of interest: The authors have declared that no conflict of interest exists.

Citation for this article: J Clin Invest. 2012;

122(3):810-813. doi:10.1172/JCI62588.
The rhythmic forward flow of blood through the major arterial vessels is controlled by coordinated electrical activation of the heart. Electrical activation in the heart originates in the sinus node, which is located in the right atrium near the entrance of the superior vena cava (1). Sinus node cells differ from normal atrial and ventricular cardiomyocytes in many respects. They develop, during embryogenesis, from precursor cells distinct from those that give rise to cardiomyocytes in the remainder of the heart. Moreover, throughout life, they maintain a molecular program that is unique and quantitatively different from that of normal atrial and ventricular cardiomyocytes (2). This molecular program endows them with unique characteristics: for example, unlike atrial and ventricular cardiomyocytes, sinus node 
A
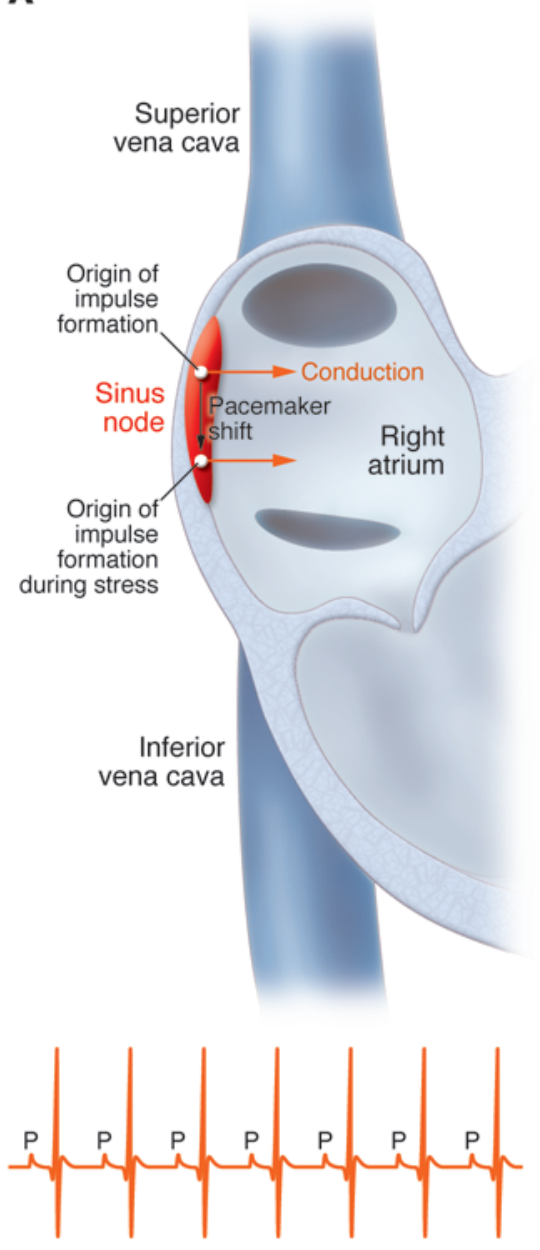

B
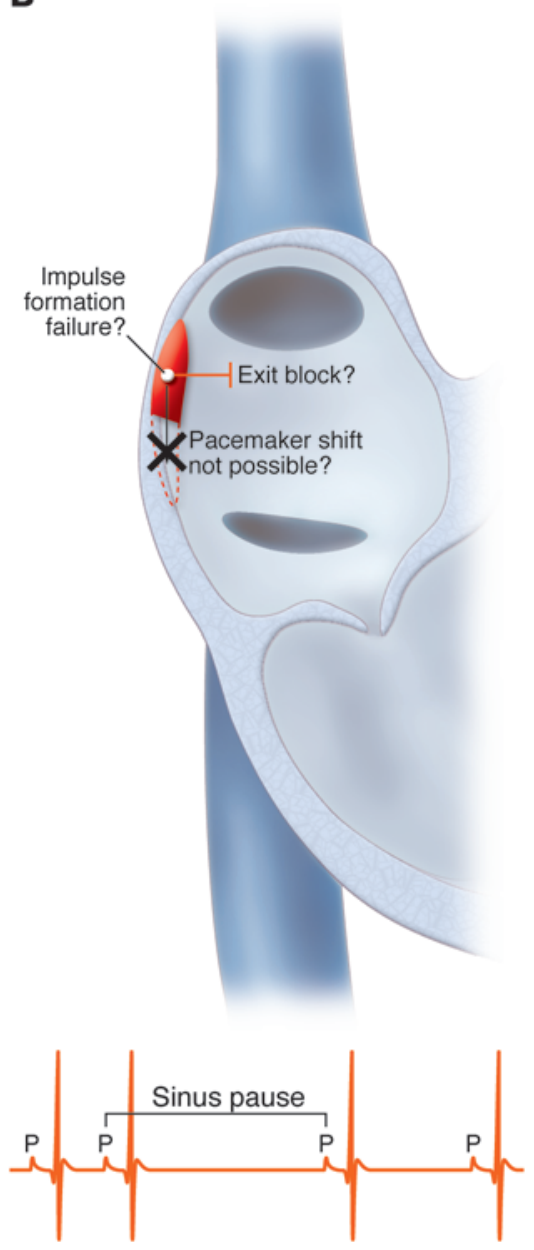

\section{Figure 1}

Possible explanations for stress-induced sinus dysfunction in mice mutant for either Popdc1 or Popdc2. (A) Wild-type sinus node and atrium. The origin of electrical impulse activation shifts upon stimulation of the $\beta$-adrenergic receptor to the inferior part of the sinus node. An electrocardiogram with normal activation of the atria is also shown. Note that the PP interval is regular. (B) Sinus node and atrium from a mouse mutant for either Popdc1 or Popdc2. The origin of electrical impulse activation cannot shift to the inferior part of the sinus node, because this part is absent. Furthermore, the sinus node has reduced in size, which may cause exit block or failure of impulse formation. An electrocardiogram during sinus node exit block or failure of impulse formation is also shown. Note that failure of atrial activation leads to an increase in PP interval (a so-called sinus pause).

cells do not have a stable resting membrane potential. In addition, the architecture of the sinus node is much more complex than that of the atrial and ventricular walls, involving numerous different cell types (3). The presence of particular gap junctions and ion channels in combination with the structure of the sinus node generates an electrophysiological environment for effective pacemaking (4).

Because the sinus node is highly innervated, and its intrinsic depolarization rate is also modulated by neurohumoral input, the rate at which the heart beats can be adjusted to physiologic requirements - it is reduced during times of rest and increased during exercise and times of stress. However, the prevalence of arrhythmias as a result of sinus node dysfunction, which affects millions of individuals later in life, indicates that pacemaking is also a vulnerable system. The most common arrhythmia related to the sinus node is sick sinus syndrome, which manifests as sinus bradycardia, atrial tachycardia, sinus arrest, or sinoatrial block. It is a common indication for implantation of a permanent electrical pacemaker (5). Changes in expression of ion channel-encoding genes, cell loss, and degenerative fibrosis are thought to be important contributors to sinus node dysfunction associated with aging (6), but the underlying molecular mechanisms are not well understood (7). In this issue of the JCI, Froese and colleagues delineate a novel molecular pathway that can cause stress-induced sinus node dysfunction in aged mice (8), a discovery that clearly has potential clinical implications. However, the paper is also an important step forward in a field that has not been studied extensively due to a lack of suitable experimental models, since it reports a new model for studying age-dependent sinus node dysfunction.

\section{Popdc1 and Popdc2 are required for stress-induced sinus node function during aging}

Popeye domain containing (Popdc) proteins contain three transmembrane helices and an evolutionary conserved cytoplasmic Popeye domain. They are expressed in heart and skeletal muscle, and their function is not known (9). Froese and colleagues showed that mice lacking either Popdc1 (also known as Bves or Pop1) or Popdc2 (also known as Pop2) are normal when housed under standard conditions but develop a striking age-dependent sinus node dysfunction when subjected to physical or mental stress (8). The mutant mice developed long periods between two subsequent atrial activations (sinus pauses) after pharmacologic $\beta$-adrenergic receptor stimulation (which mimics the effects on the heart of stress), after mental stress, and after exercise. Defective innervation and a defective response to parasympathetic stimulation (slowing heart rate) were excluded as causes of the sinus pauses, indicating an intrinsic inability of the aging mutant sinus node to respond to physiological stress or $\beta$-adrenergic receptor stimulation. Furthermore, in mutant mice, the inferior part of the sinus node was hypoplastic (Figure 1), and fewer nodal extensions into the atrium were present than in wild-type mice. Stimulation of $\beta$-adrenergic receptors causes a rise in intracellular cAMP in sinus node cells, increasing their rate of depolarization $(10,11)$. Froese and colleagues found that Popdc1 and Popdc2 constituted a novel class of high-affinity cAMP-binding proteins (8). Moreover, Popdc1 and Popdc2 were found to also bind to the stretch- 
A

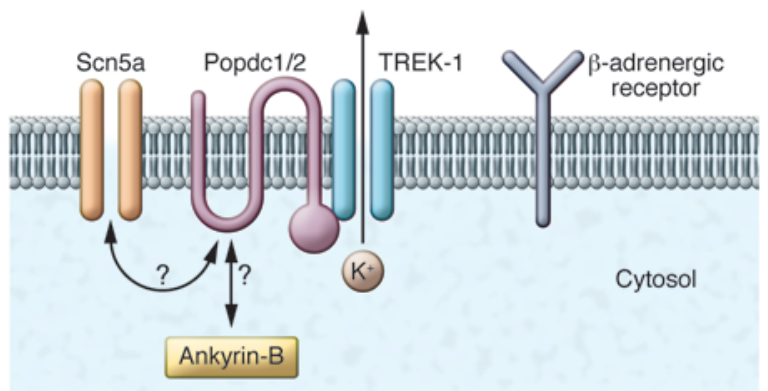

B

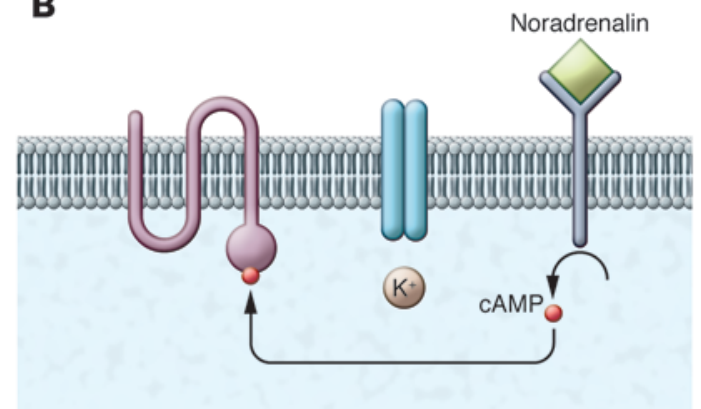

\section{Figure 2}

Mechanism of modifying TREK-1 current in rest and during stress. (A) Mechanism by which Popdc1 and Popdc2 induce a TREK-1 outward current in resting conditions, as suggested by the work of Froese and colleagues (8). Note that we believe it is possible that Popdc1 and Popdc2 also interact with Scn5a or Ankyrin-B. (B) Increased levels of cAMP induced by $\beta$-adrenergic receptor stimulation cause Popdc1 or Popdc2 to release TREK-1, resulting in inhibition of the TREK-1 outward current. This mechanism is absent in mice mutant for either Popdc1 or Popdc2, and therefore TREK-1 current is chronically reduced.

dependent potassium channel TREK-1, causing an increase in outward potassium current and thereby antagonizing pacemaker activity. Popdc1 and Popdc2 released TREK-1 upon binding cAMP (Figure 2). Thus, both $\beta$-adrenergic receptor stimulation and the absence of either Popdc1 or Popdc2 reduced outward potassium current. The elegant work of Froese and colleagues therefore demonstrates a novel and important role for Popdc1 and Popdc2 in pacemaking and provides a potentially very useful and much-needed model for agedependent sinus node dysfunction.

\section{Is it the function or the size of the sinus node?}

Sinus arrhythmia in mice mutant for either Popdc1 or Popdc 2 manifests as periods of sinus pause (8), which is the result of either a failure of impulse formation within the sinus node or a failure of impulse conduction from the sinus node to the atrium (exit block) (6). The interval of the sinus pauses was not a multiple of the normal sinus interval (8). Furthermore, the intervals between the P-waves (i.e., atrial activation) in an ECG (PP intervals) did not show a decrease prior to a skipped beat. Thus, we believe that exit block is unlikely to be the explanation for the sinus pauses observed in mice mutant for either Popdc1 or Popdc2 and that failure of impulse formation is the explanation.

Why the impulse does not form is unclear. TREK-1 channels generate an outward potassium current causing hyperpolarization of the cell membrane, which in turn makes it more difficult for a sinus node cell to reach the threshold for activa- tion. $\beta$-Adrenergic receptor stimulation increases the heart rate in part by triggering PKA-mediated phosphorylation of TREK-1, which inhibits the TREK-1 current (12). Thus, the absence of Popdc1 or Popdc2 resembles the inhibiting effect of $\beta$-adrenergic receptor stimulation on TREK-1 current. It is therefore difficult to understand how TREK-1 inhibition during $\beta$-adrenergic receptor stimulation leads to an increased rhythm, whereas TREK-1 inhibition due to absence of either Popdc1 or Popdc2 leads to sinus pauses. Hence, we suggest that the observed sinus pauses in mice mutant for either Popdc1 or Popdc2 are not solely caused by an inhibition of TREK-1 current.

$\beta$-Adrenergic receptor stimulation did not induce sinus pauses in mice mutant for either Popdc1 or Popdc2 when they were 3 months of age (8). It may be that expression of Popdc1 and Popdc 2 reduces with age; this could cause loss of redundancy, leading to sinus pauses at older ages. However, at 5 and 8 months of age, the inferior part of the sinus node was found to be hypoplastic, and fewer extensions into the atrium were present (8). Although this could be the result of dysfunction, we consider it likely that the hypoplasia of the inferior part of the sinus node contributes to the sinus pauses. If the sinus node decreases in size, it will experience a larger electrotonic load than in the normal situation. This may cause the membrane of the sinus node cells to hyperpolarize, which makes it more difficult to generate an impulse (4). Furthermore, it has been shown in other species that during $\beta$ adrenergic receptor stimulation, the origin of electrical impulse activation shifts from the center of the sinus node to the inferior part of the sinus node (13), which is the area affected in mice mutant for either Popdc1 or Popdc2 (Figure 1). This could also explain why the response to $\beta$-adrenergic receptor stimulation is blunted in these mice. However, it has also been shown that in mice, the origin of activation shifts to the superior part of the sinus node (14). To investigate how the hypoplastic inferior part of the sinus node in mice mutant for either Popdc1 or Popdc2 affects impulse formation, one could study the origin of electrical impulse activation upon $\beta$ adrenergic receptor stimulation by optical or electrical mapping. Whether TREK-1 outward current causes hyperpolarization of the membrane needs to be assessed by microelectrode recordings.

\section{Do Popdc proteins also interact with other proteins or ion channels?}

The Popdc family of proteins has never before been implicated in pacemaker activity. Froese and colleagues demonstrate that Popdc1 and Popdc 2 can specifically interact with the membrane-bound ion channel TREK-1 and that the level of cAMP modulates this interaction (8). We speculate that Popdc proteins also interact with a variety of other proteins or ion channels involved in sinus node function (Figure 2). Mutations in the genes encoding the cardiac sodium channel Nav1.5 (SCN5A) and Ankyrin-B have been described in patients with sinus arrest and sinoatrial block (15, 16). Nav1.5 forms the channels that underlie the inward sodium current that causes fast depolarization of working cardiomyo- 
cytes. An inward sodium current is also detectable in sinus node cells and is important for normal sinus node function (17, 18). Furthermore, mice with a heterozygous mutation in $\operatorname{Scn} 5$ a show sinus node dysfunction and age-dependent degeneration of sinus node tissue (19). Ankyrin-B is important for localization of ion channels within the membrane of sinus node cells. Deficiency in Ankyrin-B affects a variety of ion currents in sinus node cells, leading to sinus node dysfunction (16). Popdc proteins may thus interact with Nav1.5 and/or Ankyrin-B, thereby affecting transmembrane currents and altering sinus node function and structure. Such interactions would be affected in mice mutant for either Popdc1 or Popdc2, and this could contribute to the age-dependent sinus node dysfunction observed in these mice by Froese and colleagues.

\section{Is Popdc a new therapeutic target for treatment of sinus node dysfunction?}

Highly symptomatic patients with sinus node dysfunction are efficiently treated with implantation of a permanent electrical pacemaker (5). Screening for mutations in the POPDC1 and POPDC2 genes may help to identify people at risk for sinus node dysfunction. However, the specific mechanisms underlying sinus arrest or sinoatrial block remain poorly understood. Therefore, it would be preferable to first unravel the mechanism by which Popdc1 and Popdc2 influence the function of the sinus node. One approach could involve mutagenesis of the cAMP or TREK-1 binding site of the two Popdc proteins in mice to determine whether CAMP-mediated reduction of TREK-1 current is involved in stressinduced acceleration of sinus node rate. In addition, identifying new binding proteins for Popdc1 and Popdc2 should unravel the network in which these proteins function.

Regardless of the exact mechanism by which Popdc1 and Popdc2 function in the sinus node, Froese and colleagues convincingly demonstrate that these proteins are a novel class of membrane-localized cAMPbinding proteins that influence sinus function during aging (8). Their findings provide a new molecular mechanism involved in sinus node function under varying physiological conditions. Moreover, as age-dependent sinus node dysfunction has not been studied extensively due to the lack of suitable models, analysis of the mice mutant for either Popdc1 or Popdc2 provide a way to make much-needed inroads into studying this pathology.

\section{Acknowledgments}

We thank A.V. Postma and R. Coronel for critically reading the manuscript. This work was supported by Netherlands Heart Foundation (grant NHS 2008B062) and by the European Commission under the FP7 Integrated Project CardioGeNet (HEALTH2007-B-223463).

Address correspondence to: Vincent M. Christoffels, Heart Failure Research Center, Academic Medical Center Rm. L2-104, Meibergdreef 9, 1105 AZ Amsterdam, The Netherlands. Phone: 0031.20.5667821; Fax: 0031.20.6976177; E-mail:v.m.christoffels@ amc.uva.nl.

1. Keith A, Flack M. The form and nature of the muscular connections between the primary divisions of the vertebrate heart. J Anat Physiol. 1907; 41(pt 3):172-189.

2. Christoffels VM, Smits GJ, Kispert A, Moorman AF. Development of the pacemaker tissues of the heart. Circ Res. 2010;106(2):240-254.

3. Boyett MR, Honjo H, Kodama I. The sinoatrial node, a heterogeneous pacemaker structure. Cardiovasc Res. 2000;47(4):658-687.
4. Joyner RW, van Capelle FJL. Propagation through electrically coupled cells. How a small SA node drives a large atrium. Biophys J. 1986;50(6):1157-1164.

5. Mond HG, Proclemer A. The 11th world survey of cardiac pacing and implantable cardioverterdefibrillators: calendar year 2009--a World Society of Arrhythmia's project. Pacing Clin Electrophysiol. 2011;34(8):1013-1027.

6. Mangrum JM, DiMarco JP. The evaluation and management of bradycardia. N Engl J Med. 2000; 342(10):703-709.

7. Dobrzynski H, Boyett MR, Anderson RH. New insights into pacemaker activity: promoting understanding of sick sinus syndrome. Circulation. 2007; 115(14):1921-1932

8. Froese A, et al. Popeye domain containing proteins are essential for stress-mediated modulation of cardiac pacemaking in mice. J Clin Invest. 2012; 122(3):1119-1130.

9. Brand T. The Popeye domain-containing gene family. Cell Biochem Biophys. 2005;43(1):95-103.

10. Hammermeister KE, Yunis AA, Krebs EG. Studies on phosphorylase activation in the heart. J Biol Chem. 1965;240:986-991.

11. Toda N, Shimamoto K. The influence of sympathetic stimulation on transmembrane potentials in the S-A node. J Pharmacol Exp Ther. 1968;159(2):298-305.

12. Patel AJ, et al. A mammalian two pore domain mechano-gated S-like K+ channel. EMBO J. 1998; 17(15):4283-4290.

13. Opthof T, de Jonge B, Jongsma HJ, Bouman LN. Functional morphology of the pig sinoatrial node. J Mol Cell Cardiol. 1987;19(12):1221-1236.

14. Glukhov AV, Fedorov VV, Anderson ME, Mohler PJ, Efimov IR. Functional anatomy of the murine sinus node: high-resolution optical mapping of ankyrin-B heterozygous mice. Am J Physiol Heart Circ Physiol. 2010;299(2):H482-H491.

15 . Benson DW, et al. Congenital sick sinus syndrome caused by recessive mutations in the cardiac sodium channel gene (SCN5A). J Clin Invest. 2003;112(7):1019-1028.

16. Le SS, et al. Dysfunction in ankyrin-B-dependent ion channel and transporter targeting causes human sinus node disease. Proc Natl Acad Sci U S A. 2008;105(40):15617-15622.

17. Nathan RD. Two electrophysiologically distinct types of cultured pacemaker cells from rabbit sinoatrial node. Am J Physiol. 1986;250(2 pt 2):H325-H329.

18. Lei $\mathrm{M}$, et al. Sinus node dysfunction following targeted disruption of the murine cardiac sodium channel gene Scn5a. J Physiol. 2005;567(pt 2):387-400.

19. Hao X, et al. TGF-beta1-mediated fibrosis and ion channel remodeling are key mechanisms in producing the sinus node dysfunction associated with SCN5A deficiency and aging. Circ Arrbythm Electrophysiol. 2011;4(3):397-406 